\title{
Probing Asymmetric Charge Partitioning of Protein Oligomers during Tandem Mass Spectrometry
}

Philip D. Compton, Luca Fornelli, Neil L. Kelleher*, and Owen S. Skinner*

Departments of Chemistry and Molecular Biosciences and the Proteomics Center of Excellence, Northwestern University, Evanston, Illinois 60208, USA

\begin{abstract}
Dissociation of gaseous protein complexes produced by native electrospray often induces an asymmetric partitioning of charge between ejected subunits. We present a simple asymmetric charge partitioning factor (ACPF) to quantify the magnitude of asymmetry in this effect. When applied to monomer ejection from the cytochrome $c$ dimer and $\beta$-amylase tetramer, we found that the $\sim 60-70 \%$ of precursor charge ending up in the ejected monomers corresponds to ACPFs of 1.38 and 2.51, respectively. Further, we used site-specific fragmentation from electron transfer dissociation (ETD) to identify differences in fragmentation and characterize domains of secondary-structure present in the dimer, ejected monomers, and monomers obtained directly from electrospray ionization (ESI). We found evidence of structural changes between the dimer and ejected monomer, but also that the ejected monomer had a nearly identical set of fragment ions produced by ETD as the ESI monomer with the same charge state. Surprisingly, APCF values for ETD fragment ions generated directly from the dimer revealed that the fragments undergo asymmetric charge partitioning at over twice the magnitude of that observed for ejection of the monomer.
\end{abstract}

Keywords: protein complexes; proteomics; electron transfer dissociation; asymmetric charge partitioning

*Email: n-kelleher@northwestern.edu

*Email: OwenSkinner2012@u.northwestern.edu 


\section{Introduction}

Native electrospray ionization (ESI) coupled with mass spectrometry has proven extremely useful for the analysis of large assemblies of biological macromolecules. ${ }^{1,2}$ Because many non-covalent interactions are maintained during ionization, it is possible to study protein-protein ${ }^{3,4}$, and protein-ligand ${ }^{5}$ complexes by mass spectrometry (MS) that would be destroyed under denaturing conditions. The stoichiometry of these macromolecular assemblies plays a critical role in many cellular processes ${ }^{6}$, including disease states $^{7}$, making the direct observation of an intact complex of high interest in the study of biological and gas-phase phenomena alike.

Beyond high-accuracy intact mass measurement, gas-phase fragmentation can provide further identification and characterization of the intact complex ${ }^{8,9}$ as well as individual subunits. ${ }^{10,11}$ In addition, fragmentation using electron capture dissociation (ECD) can cleave covalent protein backbone bonds while maintaining nearby non-covalent interactions. ${ }^{12}$ Recent studies have utilized this unique property to provide information about the higher-order structure of gaseous proteins ${ }^{13-15}$ and protein complexes. ${ }^{8,16}$ Similarly, native electron capture dissociation (NECD), a new sub-type of radical dissociation that is induced by heating the transfer capillary instead of the addition of exogenous electrons, was used to probe the gas-phase unfolding of the cytochrome $c$ dimer. ${ }^{17,18}$

On the other hand, threshold dissociation of protein complexes by collisional or photon-based activation often liberates one or more monomers with a disproportionately high charge, in a process first described by Light-Wahl, et al. in $1994 .{ }^{19}$ The effect has been studied by many labs since, and termed "Asymmetric Charge Partitioning" (ACP). ${ }^{20-22}$ It can be attributed to, among other reasons, the unfolding of one monomer prior to or during ejection, causing it to acquire far more charge than would be expected based on its portion of the mass of the intact complex. ${ }^{23}$ Studies by the Robinson group have further showed a close correlation between the ratio of surface areas of the ejected monomer and the intact complex and the overall degree of charge transfer. ${ }^{24}$ Experimental results from several labs on a variety of systems ${ }^{25}$ have also reproduced the effect and found ACP to be dissociation method ${ }^{26}$ and sometimes charge state ${ }^{23}$ dependent. Theoretical studies have also been performed, using molecular dynamics ${ }^{27}$ and other modeling methods. ${ }^{28}$

Here, we introduce a simple metric for quantifying $A C P$, and use electron transfer dissociation (ETD) ${ }^{29}$, a radical fragmentation technique similar to $E C D$, to probe the secondary structures in the gas-phase cytochrome $c$ dimer, its ejected monomer, and the analogous monomer produced via electrospray. We not only find evidence of the structural changes between the dimer and ejected monomer, but also of an ACP upon dissociation of the fragment ions formed by ETD. This observation provides the first characterization of ACP as a general phenomenon for any sub-structural element liberated from a protein complex.

\section{Material and Methods}

Horse heart cytochrome $c$ was purchased from Sigma Aldrich (St. Louis, MO, USA) and used without further purification. Cytochrome $c$ samples were sprayed at $5 \mu \mathrm{L} / \mathrm{min}$ from a $0.26 \mathrm{mg} / \mathrm{mL}$ solution in 100 $\mathrm{mM}$ ammonium acetate on an Orbitrap Elite mass spectrometer (Thermo Scientific, Bremen, Germany). 
$\beta$-amylase samples were purchased from Sigma Aldrigh (St. Louis, MO, USA) and were buffer exchanged into $100 \mathrm{mM}$ ammonium acetate using $30 \mathrm{kDa}$ molecular weight cutoff filters (Millipore, Billerica, MA, USA). Samples were sprayed and analyzed with a modified Q-Exactive HF mass spectrometer (Thermo Scientific, Bremen, Germany) as described previously ${ }^{10}$.

ETD was performed with fluoranthene as the reagent using an $\mathrm{N}_{2}$ carrier gas. When ETD was performed for precursor charge reduction without fragmentation (ETnOD), ${ }^{30}$ the reaction time was increased (generally $>5 \mathrm{~ms}$ ) to maximize the signal of the targeted reduced-charge species. When ETD was performed for backbone cleavage, ETD reaction times were set to maximize fragment ion production while minimizing reduced precursor ion signal, generally 2-10 ms depending on the original precursor charge state. CAD indicates RF-induced collisional activation and was performed in an ion trap. CAD was used to produce the ejected monomer as well as to break non-covalent bonds after ETnoD (i.e. supplemental activation) in selected experiments. All data were analyzed manually with the freely available software, mMass. ${ }^{31}$ Ion yields were normalized by charge to account for the linear detection bias in the Orbitrap analyzer. ${ }^{32}$

\section{Results and Discussion:}

\subsection{Monomer Ejection from the Dimer of Cytochrome $c$}

Fig. 1 depicts spectra of cytochrome $c$ monomers ejected from dimeric precursors of selected charge states. In the top panel, the 13+ charge state of horse heart cytochrome $c$ dimer was isolated and activated with CAD to eject monomer ions of charge states $10+-6+$. To prevent overlap of dimer and monomer signals, the isolated $13+$ dimer was subjected to ETD at reaction times optimized for formation of the reduced-charge precursors that have not fragmented (ETnoD). ${ }^{30}$ Each of the $12+-9+$ reduced-charge dimer species were then isolated individually, and activated with CAD to eject monomers. No significant yields of $\boldsymbol{c}$ - or $\boldsymbol{z}$-fragment ions were observed relative to signals from monomer ejection. Each dimer charge state is therefore a product of the parent $13+$ ion, however, isolation and activation of the ESI-formed 11+ ion produced similarly-charged ejected monomers (Supplementary Fig. 1).

Fig. 2 shows the intensity-weighted average charge of ejected monomers resulting from each dimer charge state (black diamonds). The green bars underneath indicate the percent of the precursor charge represented by the weighted-average monomer charge. Despite the general downward trend of ejected monomer charge (Fig. 2), the overall percent remains remarkably constant, varying from $66-72 \%$. Thus, the magnitude of ACP does not change even with a 31\% decrease in precursor charge density, indicating that it is more affected by the relative unfolding of the two subunits than it is by the Coulombic strain of charges on the precursor for the 13-9+.

Monomer charge states from all of the ejected dimers show significant evidence of ACP. A previous study by Williams and co-workers ${ }^{23}$ found significant differences between the monomer ejection from cytochrome $c$ dimers formed directly by ESI when compared to those formed by gas-phase deprotonation of isolated higher charge states. Specifically, they found that when the $15+$ cytochrome $c$ dimer is deprotonated to form the $13+$, it produces symmetrically-ejected monomers. The current results do not show any evidence of this effect: the doubly-reduced $11+$ dimers produced nearly 
identical ejected monomers when compared to those produced from the ESI-formed 11+. The apparent discrepancy in results can be attributed to several factors, including the higher pressure of the ion trap ( $\sim 4$ mTorr) when compared to that found in an ICR instrument and the difference in proton transfer/electron transfer reagent (diethylamine instead of the fluoranthene used here). The magnitude of ACP can also vary greatly for complexes electrosprayed out of different solution conditions and at different concentrations; a previous study by Smith and co-workers ${ }^{33}$ showed no ACP with dissociation of cytochrome $c$ dimers under denaturing conditions. Indeed, the full mass spectrum of cytochrome $c$ prior to isolation and dissociation (Supplementary Fig. 2) shows monomer species of relatively high charge $(12+-10+)$ indicating some degree of solution denaturation.

\subsection{Asymmetric Charge Partitioning Factor}

In order to formalize the magnitude of $A C P$, we introduce the asymmetric charge partitioning factor (ACPF, Eq. 3.2.1), which can be used to compare ACP across multiple systems with different numbers of subunits.

$$
\text { Eq.3.2.1 } \quad A C P F=\frac{C_{E S} M_{I}}{C_{I} M_{E S}}
$$

Here, $C_{E S}$ and $C_{1}$ indicate the intensity-weighted average charges of the ejected subunits and the intact complex, respectively, while $M_{E S}$ and $M_{1}$ indicate the masses of the ejected subunit and complex, respectively. Essentially, the ACPF compares the average charge present on observed ejected subunits with the theoretical charge that would be present following a symmetric partitioning of charge from the precursor protein complex. A completely symmetric charge partitioning would be indicated by an ACPF of 1 , with higher values indicating increasingly asymmetric charge partitioning. Note that because this factor uses the intensity-weighted average charge of the ejected monomers, $m / z$-dependent biases in measuring intensity will affect APCF values.

The example of the $13+$ cytochrome $c$ dimer mentioned in section 3.1 produces an intensity-weighted average ejected monomer charge of 8.66 (Figs. 1 and 2), corresponding to an ACPF of 1.33. Monomer ejection from the other dimer charge states produced product ions with a similar percent of the total precursor charge, thus their ACPF values are also similar (1.33-1.43).

In order to show its application to a variety of systems, we use the ACPF to examine monomer ejection of the $224 \mathrm{kDa}$ homotetrameric $\beta$-amylase purified from sweet potatoes. The ejected monomers from the quadrupole-isolated $32+, 31+$ and $30+$ intact tetramers are shown in Fig. 3 . Heterogeneity in the monomer mass can be attributed to differing numbers of hexose subunits (multiples of $162 \mathrm{Da}$ ), likely due to differential O-glycosylation. The calculated ACPFs range from 2.51-2.52 (Fig. 3), again appearing to be little affected by the precursor charge. Thus, we can directly compare the magnitude of ACP in $\beta$ amylase with that of cytochrome $c$ dimer by using the ACPF, indicating a nearly two-fold stronger charge partitioning effect in the tetramer. Higher ACP has previously been described for monomer ejection from tetramers when compared with that from dimers, and has been attributed to the change in the ratios of surface areas between the two species ${ }^{24}$.

\subsection{ETD Fragmentation}


ETD fragment ion yields from the 13+ cytochrome $c$ dimer are plotted with respect to their cleavage sites in Fig. 4a. Here, $\boldsymbol{c}$ and $z$ fragment yields (both even- and odd-electron species were observed from both fragment types) are summed together. All yields are normalized to the base peak intensity. Cleavage products are plotted in the same way for the 9+ ejected monomer in Fig. 4b. On the other hand, Fig. 4c shows electron-based cleavage products for the ejected monomer after ETD, followed by isolation of the singly-reduced $(\mathrm{M}+9 \mathrm{H})^{8+\bullet}$ ETnoD molecular ion, and further subjected to low-energy CAD to release electron transfer fragment ions that had not completely dissociated. Although low abundance $\boldsymbol{b}$ - and $\boldsymbol{y}$-fragments were observed, they were not considered as they likely occurred during the CAD process.

Fragmentation yields from the dimer appear to exhibit similar relative abundances when compared to those from the ejected monomer (e.g. yields from backbone cleavage sites 28,41 , and 42). However, the dimer undergoes increased fragmentation around site 37, and its most abundant peak, from site 79, is not present in the monomer. The presence of additional fragmentation channels in the dimer is consistent with a different set of cytochrome $c$ conformers that are not present in the ejected monomer. Further, the dimer form of cytochrome $c$ does not exist under biological conditions, contributing to increased heterogeneity in the dimer fold. It is possible that the other monomermonomer contacts in the dimer provide additional stabilization for conformers that are unstable by themselves. Additional fragmentation from the dimer is also remarkably similar to that found after native electron capture dissociation ${ }^{17}$, which produced the most abundant fragmentation from cleavage site 79 as well.

Neither the dimer nor the ejected monomer produced substantial fragmentation from the C-terminal region, with significant fragment yields only occurring in the ejected monomer after ETnoD and subsequent activation with CAD (Fig. 4c). Similar to previous experiments that followed ECD with activation by infrared photons, ${ }^{13}$ the additional fragment ions formed from gentle CAD indicate that it is a region with significant higher-order structure where the products from backbone cleavage events are prevented from dissociation by non-covalent interactions. These results are consistent with gas-phase salt bridging in that region, predicted to be one of the strongest non-covalent interactions in the gas phase. $^{34}$

Product ion yields from the ESI-produced 9+ monomer after ETD (Fig. 4d) and after ETnoD/CAD (Fig. 4e) exhibit a remarkable similarity to those produced from the ejected monomer (Fig. 4b and 4c). The relative abundances of ETD products, as well as the regions that are fragmented are nearly identical between the two, indicating similarities in both secondary and tertiary protein structure. The similar protein structures of both the ejected monomer and the ESI-produced molecular ions can be attributed to rapid gas-phase rearrangement of either the protein structure or the spatial distribution of protons in the relatively high pressure ( $\sim 4 \mathrm{mTorr} \mathrm{He})$ in the ion trap. The rearrangement of conformers at higher pressures was also observed with the charge-reduced dimers and discussed in section 3.1.

Mapping the dimer ETD fragment ion yields onto the crystal structure of monomeric cytochrome $c$ (Fig. 5a) indicates increased dimer ETD cleavage in the disordered region (residues 30-40) at the bottom center of the figure when compared to that of the ejected monomer (Fig. 5b). On the other hand, cleavage products from the ejected monomer after ETnoD/CAD (Fig. $5 \mathrm{c}$ ) result mostly from the Cterminal $\alpha$-helix. Whether $\alpha$-helices are retained in the gas phase, and whether they inhibit electronbased cleavage is still a matter of debate. ${ }^{35-37}$ However, the apparent refolding of both the ESI-produced 
and ejected monomers to this state indicates favorable formation of higher-order structures in this domain.

\subsection{Asymmetric Charge Partitioning of ETD Fragment lons}

The intensity-weighted average charge of site-specific ETD fragmentation products originating from the intact dimer, the ejected monomer, and the ejected monomer after ETnoD/CAD are shown in Fig. 6a. The two $z$-fragments observed from the dimer were not included; for those from the ejected monomer, the complementary charge was used assuming one proton was neutralized during fragmentation $(8-z$ fragment charge). Therefore, the charge value indicates the average number of charges found on the $\mathrm{N}$ terminal cleavage product of a fragmentation event.

Determination of the ACPF described in section 3.2 for each fragmentation product is shown in Fig. $6 \mathrm{~b}$. Here, fragmentation from both ETD and ETnoD/CAD of the ejected monomer are considered together. Unlike in Fig. 6a, the charge of fragment ions here are considered to contain the charge neutralized during the ETD process, and the monomer and dimer charges are considered before neutralization (9+ and $13+$, respectively). For simplicity, the mass of each fragment is determined by the number of residues, instead of the molecular mass of the fragment. A solid horizontal line at ACPF $=1$ is shown to indicate symmetric charge partitioning, while the dashed line at ACPF $=1.3$ indicates the magnitude of ACP during monomer ejection from the $13+$ dimer.

Despite the $28 \%$ decrease of charge per residue of the dimer when compared to the ejected monomer, many of the dimer's fragment ions dissociate with a higher charge than those from the monomer. The corresponding ACPF of these fragment ions accounts for these factors and indicates the strength of the effect: at cleavage site 28, fragments dissociate with an ACPF of 2.8, more than double that observed for the same ETD fragment ions generated from the ejected monomer. While the ACPF trends lower at larger fragment sizes, it remains $\sim 1$ higher than that from fragments of the ejected monomer, indicating a significant increase in the effect. A similar effect was also briefly described for native ECD fragments. ${ }^{17}$

In order to determine the portion of the ACP that can be attributed to differential charging of the iron atom in the heme group, we examined the deconvoluted ion mass of the $\boldsymbol{c}_{28}$ fragment ion at both 2+ and $3+$ from the dimer, the ejected monomer, and the ESI-produced monomer (Supplementary Fig. 3 ). Fragment masses correspond to the unambiguous addition of a proton between the $2+$ and $3+$ from all of the precursors, indicating that the observed changes in charge state are due to protonation, instead of oxidation of the heme iron. The large ACPF can therefore be directly attributed to proton transfer.

We propose two potential causes for the increased ACPF in ETD product ions: either the charges have asymmetrically partitioned prior to ETD fragmentation, or the charges are transferred after fragmentation and before ejection from the complex. The Coulombic repulsion between protons makes the former unlikely. In the latter, we postulate that the electron transfer event occurs almost instantaneously, cleaving the protein backbone. This is characteristic of a non-ergodic process. ${ }^{12}$ However, these fragment ions do not dissociate immediately, and remain attached to the protein complex through non-covalent interactions. Collisions with residual gas in the ion trap gently activate the fragment-dimer complex causing the fragment to elongate at which point additional charge is transferred in a similar process to that of monomer ejection. Charge transfer at this stage must either occur through space, or through the non-covalent interactions between the fragment and the complex; the covalent backbone bond has already been broken. The mobilized charges, likely driven to regions 
with the lowest charge density, account for the gradual increase in charge found for cleavage sites 28-60 in Fig. 6a. When the c-type fragment ion eventually leaves the complex, the process is highly asymmetrical and creates products with high charge density where ACPF is $>1$ (Fig. 6b).

\section{Conclusions}

We introduced a simple metric, the asymmetric charge partitioning factor, or ACPF, to quantify the extent of charge partitioning occurring as protein complexes break apart in the gas phase. Using the ACPF metric, we found that ACP of the cytochrome $c$ dimer and the $\beta$-amylase tetramer are not dependent on precursor charge when dissociated with CAD at ion-trap pressures. Further, ETD product ions indicate a similar, but not identical higher-order structure of the intact dimer and ejected monomer, and that the ejected monomer has undergone new folding. Surprisingly, ETD products from the dimer are observed with higher charge, and exhibit a higher ACPF than those from the monomer, which has a higher charge-density precursor. Thus, we provide compelling evidence that ETD products not only undergo ACP, but can absorb even more charge density than the ejected monomer when normalized for their mass. Future studies can test this effect on other systems and other solution conditions and refine fragment-ion specific surface area models for better understanding and use of the diverse tandem MS methods in structural biology.

\section{Acknowledgements:}

This work was financially supported by the W. M. Keck foundation. LF is funded through an Early Postdoc Mobility Fellowship from the Swiss National Science Foundation. OSS would like to thank the NSF GRFP for a predoctoral fellowship (\#2014171659) and NLK acknowledges the NIH (GM067193) for further support of this work.

\section{TOC Figure:}
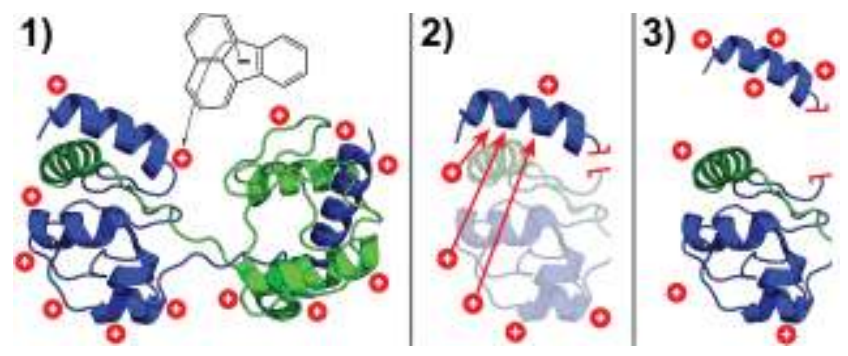


\section{Figure Captions:}

Figure 1. Ejected monomer species observed from isolation of the $13+$ through $9+$ species of the cytochrome $c$ dimer (precursor charge state listed at far left). The $12+$ to $9+$ parent ions (indicated by an asterisk) were formed by charge reduction (ETnoD) of the 13+ during the ETD process.

Figure 2. Black diamonds indicate the intensity-weighted average charge of the ejected monomers (left axis) observed after isolation and fragmentation of each dimer charge state ( $x$-axis). The green bars represent the percent of charge from the dimeric precursor that is observed in each monomer for each monomer ejection experiment (right axis).

Figure 3. Monomers ejected in the gas phase from isolated charge states of the homotetrameric complex $\beta$-amylase; charge states of the precursor tetramer are indicated by the numbers at far left, ACPF values are indicated by numbers on far right.

Figure 4. Site-specific ETD product ion abundances (sum of even- and odd-electron $\boldsymbol{c}$ - and $z$-fragment ions) from each cleavage site observed from the following five precursors and experiments: (a) ETD of the intact 13+ dimer of cytochrome $c$, (b) ETD of the 9+ ejected monomer, (c) ETnoD of the 9+ ejected monomer with additional activation by gentle CAD to break non-covalent interactions, (d) ETD of the 9+ ion monomer obtained directly from electrospray, and (e) ETnoD of the electrosprayed 9+ ion of the monomer followed by gentle CAD. All fragment ions abundances are normalized to that of the base peak, which is set to 1 .

Figure 5. Mapping ETD fragment ion yields onto the crystal structure of monomeric cytochrome $c$ for (a) the dimer, (b) the ejected monomer, and (c) the ejected monomer after ETD, isolation of the singly reduced (ETnoD) molecular ion, and gentle activation by CAD. All fragment yields were normalized to the most abundant peak of the spectrum, which was set to 1 ; red indicates a range of $0.1-1$, orange: 0.03-0.1, yellow: <0.03, white: no fragments observed.

Figure 6. (a) The intensity-weighted average charge of fragment ions observed from each cleavage site for ETD products originating from the intact dimer, the ejected monomer, and the ejected monomer after ETnoD/CAD of cytochrome $c$ (see key for designations). (b) The asymmetric charge partitioning factor (APCF) calculated for each fragment ion. The dashed horizontal line indicates the average value for monomer ejection; an APCF value of 1 (i.e., symmetric charge partitioning) is indicated with the solid horizontal line. Only fragment ions with cleavage sites $>20$ are considered. 


\section{References:}

(1) Yang, Y.; Barendregt, A.; Kamerling, J. P.; Heck, A. J. R. Analytical Chemistry 2013, 85, 12037.

(2) Muneeruddin, K.; Thomas, J. J.; Salinas, P. A.; Kaltashov, I. A. Analytical Chemistry 2014, $86,10692$.

(3) Loo, J. A. Mass Spectrom. Rev. 1997, 16, 1.

(4) Barrera, N. P.; Di Bartolo, N.; Booth, P. J.; Robinson, C. V. Science 2008, 321, 243.

(5) Xie, Y.; Zhang, J.; Yin, S.; Loo, J. A. J. Am. Chem. Soc. 2006, 128, 14432.

(6) Gavin, A. C.; Bosche, M.; Krause, R.; Grandi, P.; Marzioch, M.; Bauer, A.; Schultz, J.; Rick, J. M.; Michon, A. M.; Cruciat, C. M.; Remor, M.; Hofert, C.; Schelder, M.; Brajenovic, M.; Ruffner, H.; Merino, A.; Klein, K.; Hudak, M.; Dickson, D.; Rudi, T.; Gnau, V.; Bauch, A.; Bastuck, S.; Huhse, B.; Leutwein, C.; Heurtier, M. A.; Copley, R. R.; Edelmann, A.; Querfurth, E.; Rybin, V.; Drewes, G.; Raida, M.; Bouwmeester, T.; Bork, P.; Seraphin, B.; Kuster, B.; Neubauer, G.; Superti-Furga, G. Nature 2002, 415, 141.

(7) Tefferi, A.; Lasho, T. L.; Abdel-Wahab, O.; Guglielmelli, P.; Patel, J.; Caramazza, D.; Pieri, L.; Finke, C. M.; Kilpivaara, O.; Wadleigh, M.; Mai, M.; McClure, R. F.; Gilliland, D. G.; Levine, R. L.; Pardanani, A.; Vannucchi, A. M. Leukemia 2010, 24, 1302.

(8) Zhang, H.; Cui, W.; Wen, J.; Blankenship, R. E.; Gross, M. L. Journal of the American Society for Mass Spectrometry 2010, 21, 1966.

(9) Barrera, N. P.; Isaacson, S. C.; Zhou, M.; Bavro, V. N.; Welch, A.; Schaedler, T. A.; Seeger, M. A.; Miguel, R. N.; Korkhov, V. M.; van Veen, H. W.; Venter, H.; Walmsley, A. R.; Tate, C. G.; Robinson, C. V. Nat. Methods 2009, 6, 585.

(10) Belov, M. E.; Damoc, E.; Denisov, E.; Compton, P. D.; Horning, S.; Makarov, A. A.; Kelleher, N. L. Anal Chem 2013, 85, 11163.

(11) Skinner, O. S.; Do Vale, L. H. F.; Catherman, A. D.; Havugimana, P. C.; Sousa, M. V. d.; Compton, P. D.; Kelleher, N. L. Analytical Chemistry 2015, 87, 3032.

(12) Zubarev, R. A.; Kelleher, N. L.; McLafferty, F. W. J. Am. Chem. Soc. 1998, 120, 3265.

(13) Breuker, K.; Oh, H.; Horn, D. M.; Cerda, B. A.; McLafferty, F. W. J. Am. Chem. Soc. 2002, $124,6407$.

(14) Skinner, O. S.; McLafferty, F. W.; Breuker, K. J Am Soc Mass Spectrom 2012, 23, 1011.

(15) Skinner, O.; Breuker, K.; McLafferty, F. J. Am. Soc. Mass Spectrom. 2013, $24,807$.

(16) Li, H.; Wongkongkathep, P.; Van Orden, S. L.; Ogorzalek Loo, R. R.; Loo, J. A. J Am Soc Mass Spectrom 2014, 25, 2060.

(17) Breuker, K.; McLafferty, F. W. Angewandte Chemie International Edition 2003, 42, 4900.

(18) Breuker, K.; McLafferty, F. W. Angewandte Chemie (International ed. in English) 2005, $44,4911$.

(19) Light-Wahl, K. J.; Schwartz, B. L.; Smith, R. D. Journal of the American Chemical Society 1994, 116, 5271.

(20) Schwartz, B.; Bruce, J.; Anderson, G.; Hofstadler, S.; Rockwood, A.; Smith, R.; Chilkoti, A.; Stayton, P. Journal of the American Society for Mass Spectrometry 1995, 6, 459. 
(21) Jurchen, J. C.; Williams, E. R. Journal of the American Chemical Society 2003, 125, 2817.

(22) Rostom, A. A.; Robinson, C. V. Current Opinion in Structural Biology 1999, 9, 135.

(23) Jurchen, J. C.; Garcia, D. E.; Williams, E. R. Journal of the American Society for Mass Spectrometry 2004, 15, 1408.

(24) Benesch, J. L. P.; Aquilina, J. A.; Ruotolo, B. T.; Sobott, F.; Robinson, C. V. Chemistry \& Biology 2006, 13, 597.

(25) Abzalimov, R. R.; Frimpong, A. K.; Kaltashov, I. A. International Journal of Mass Spectrometry 2006, 253, 207.

(26) Jones, C. M.; Beardsley, R. L.; Galhena, A. S.; Dagan, S.; Cheng, G.; Wysocki, V. H. Journal of the American Chemical Society 2006, 128, 15044.

(27) Wanasundara, S. N.; Thachuk, M. Journal of the American Society for Mass Spectrometry 2007, 18, 2242.

(28) Sciuto, S.; Liu, J.; Konermann, L. Journal of The American Society for Mass Spectrometry 2011, 22, 1679.

(29) Syka, J. E. P.; Coon, J. J.; Schroeder, M. J.; Shabanowitz, J.; Hunt, D. F. Proc. Natl. Acad. Sci. U. S. A. 2004, 101, 9528.

(30) Sadygov, R. G.; Good, D. M.; Swaney, D. L.; Coon, J. J. Journal of proteome research $2009,8,3198$.

(31) Strohalm, M.; Kavan, D.; Novák, P.; Volný, M.; Havlíček, V. r. Analytical Chemistry 2010, 82,4648 .

(32) Hamidane, H.; He, H.; Tsybin, O.; Emmett, M.; Hendrickson, C.; Marshall, A.; Tsybin, Y. Journal of the American Society for Mass Spectrometry 2009, 20, 1182.

$27,811$.

(33) Smith, R. D.; Light-Wahl, K. J.; Winger, B. E.; Loo, J. A. Organic Mass Spectrometry 1992,

(34) Prell, J. S.; Demireva, M.; Oomens, J.; Williams, E. R. Journal of the American Chemical Society 2009, 131, 1232.

(35) Breuker, K.; Bruschweiler, S.; Tollinger, M. Angewandte Chemie (International ed. in English) 2011, 50, 873.

(36) Wyttenbach, T.; Bowers, M. T. The Journal of Physical Chemistry B 2011, 115, 12266.

(37) Breuker, K.; McLafferty, F. W. Proceedings of the National Academy of Sciences of the United States of America 2008, 105, 18145. 


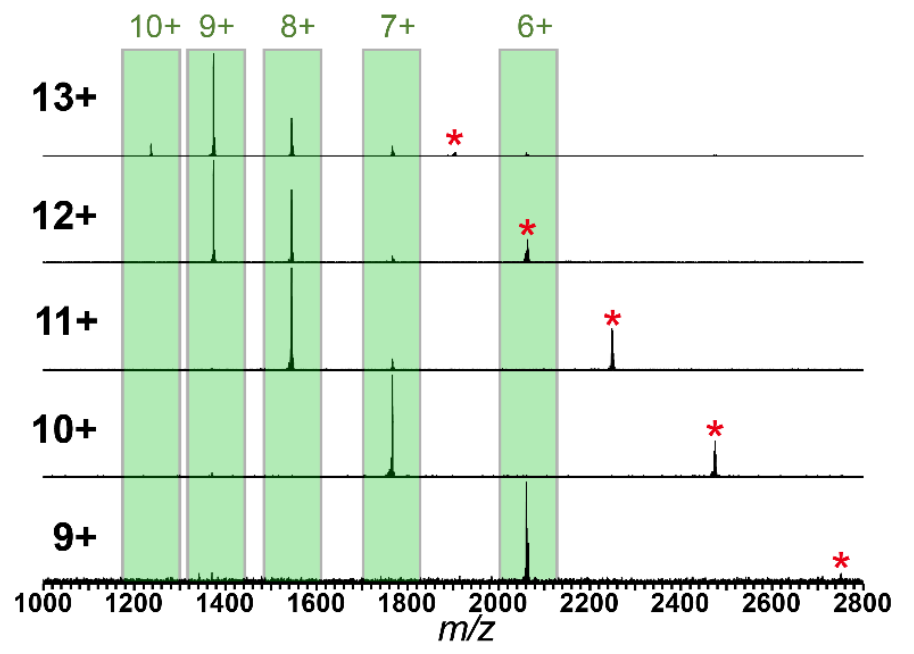

Figure 1. Ejected monomer species observed from isolation of the $13+$ through $9+$ species of the cytochrome $c$ dimer (precursor charge state listed at far left). The $12+$ to $9+$ parent ions (indicated by an asterisk) were formed by charge reduction (ETnoD) of the 13+ during the ETD process.

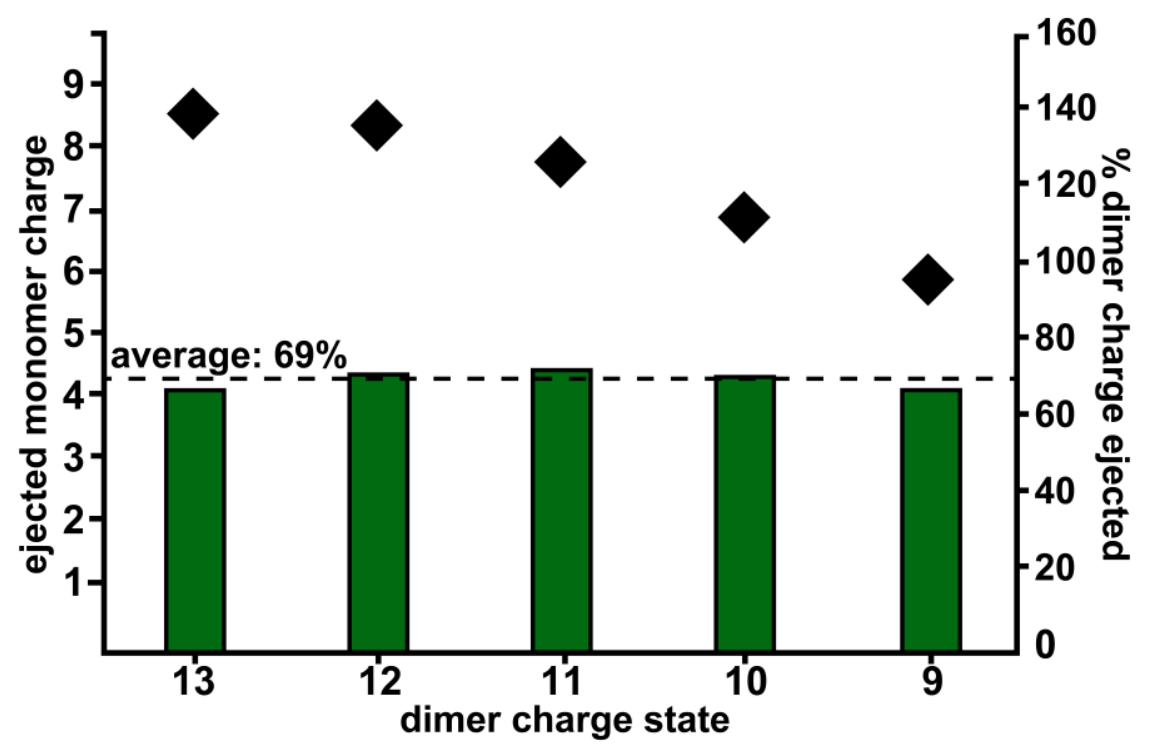

Figure 2. Black diamonds indicate the intensity-weighted average charge of the ejected monomers (left axis) observed after isolation and fragmentation of each dimer charge state ( $x$-axis). The green bars 
represent the percent of charge from the dimeric precursor that is observed in each monomer for each monomer ejection experiment (right axis).

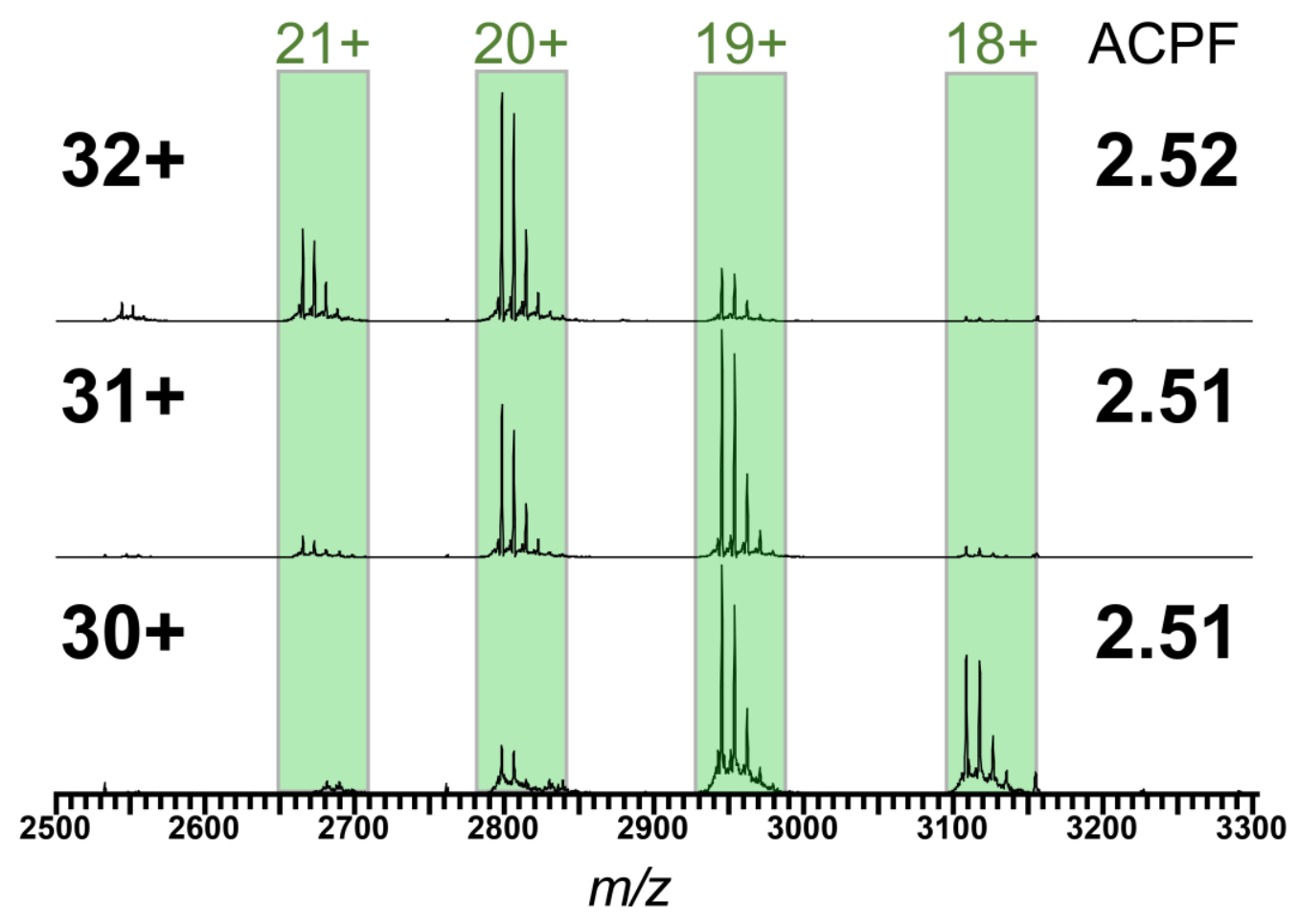

Figure 3. Monomers ejected in the gas phase from isolated charge states of the homotetrameric complex $\beta$-amylase; charge states of the precursor tetramer are indicated by the numbers at far left, ACPF values are indicated by numbers on far right. 


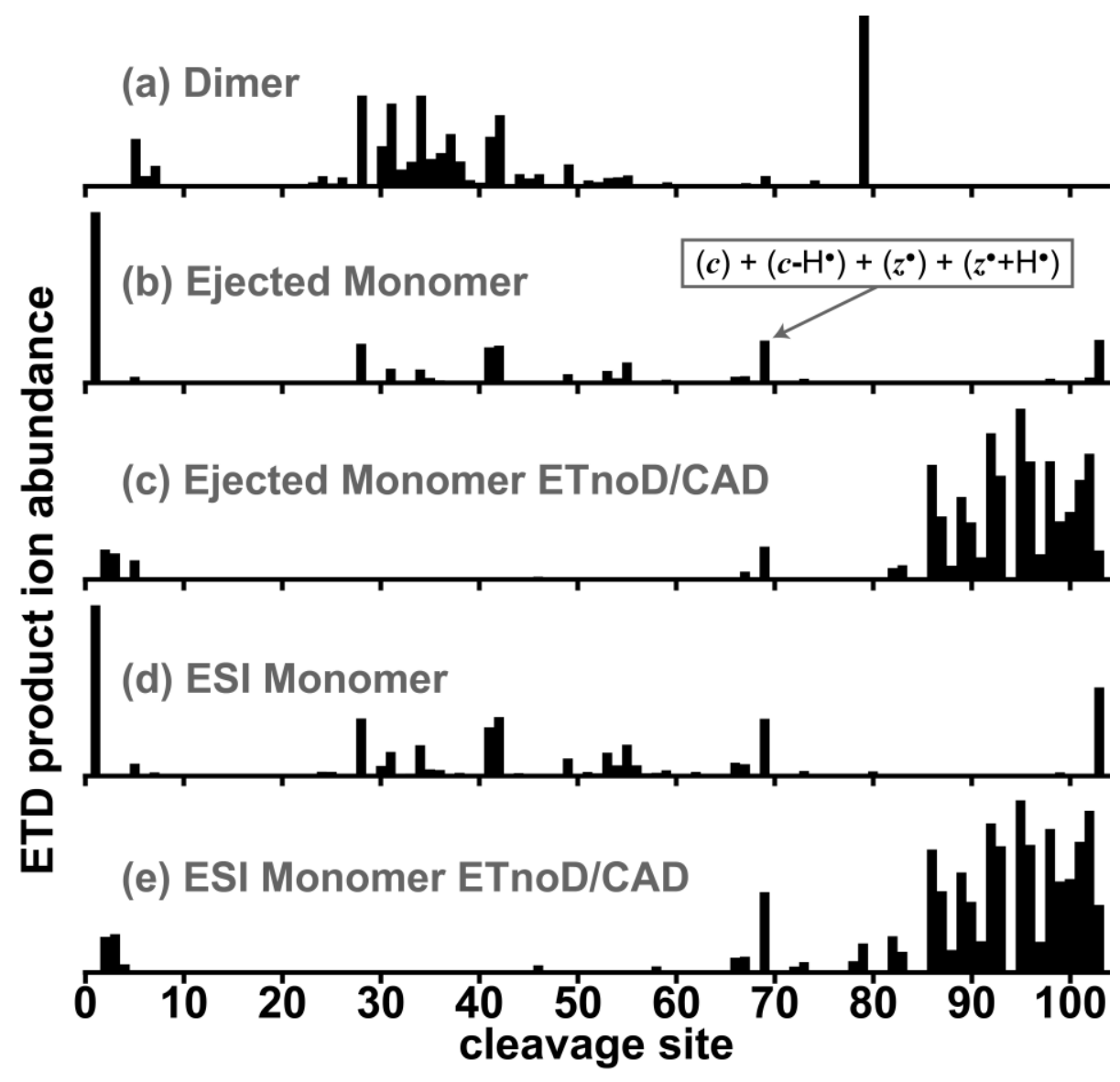

Figure 4. Site-specific ETD product ion abundances (sum of even- and odd-electron $\boldsymbol{c}$ - and $z$-fragment ions) from each cleavage site observed from the following five precursors and experiments: (a) ETD of the intact 13+ dimer of cytochrome $c$, (b) ETD of the 9+ ejected monomer, (c) ETnoD of the 9+ ejected monomer with additional activation by gentle CAD to break non-covalent interactions, (d) ETD of the 9+ ion monomer obtained directly from electrospray, and (e) ETnoD of the electrosprayed 9+ ion of the monomer followed by gentle CAD. All fragment ions abundances are normalized to that of the base peak, which is set to 1 .
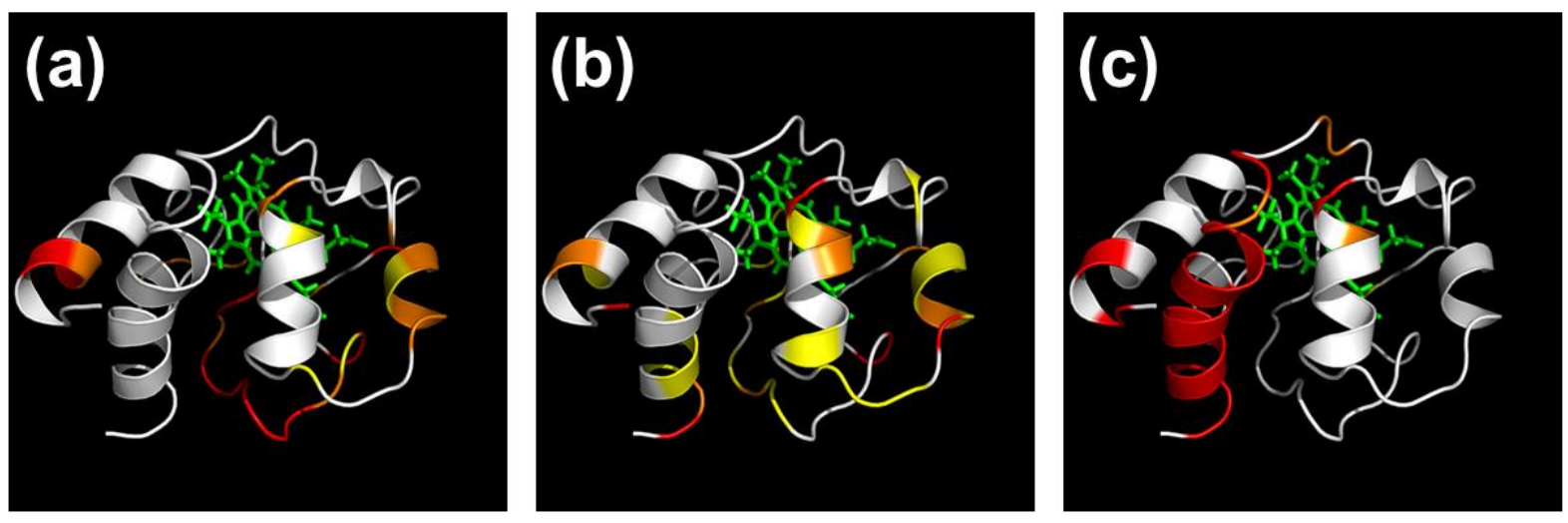

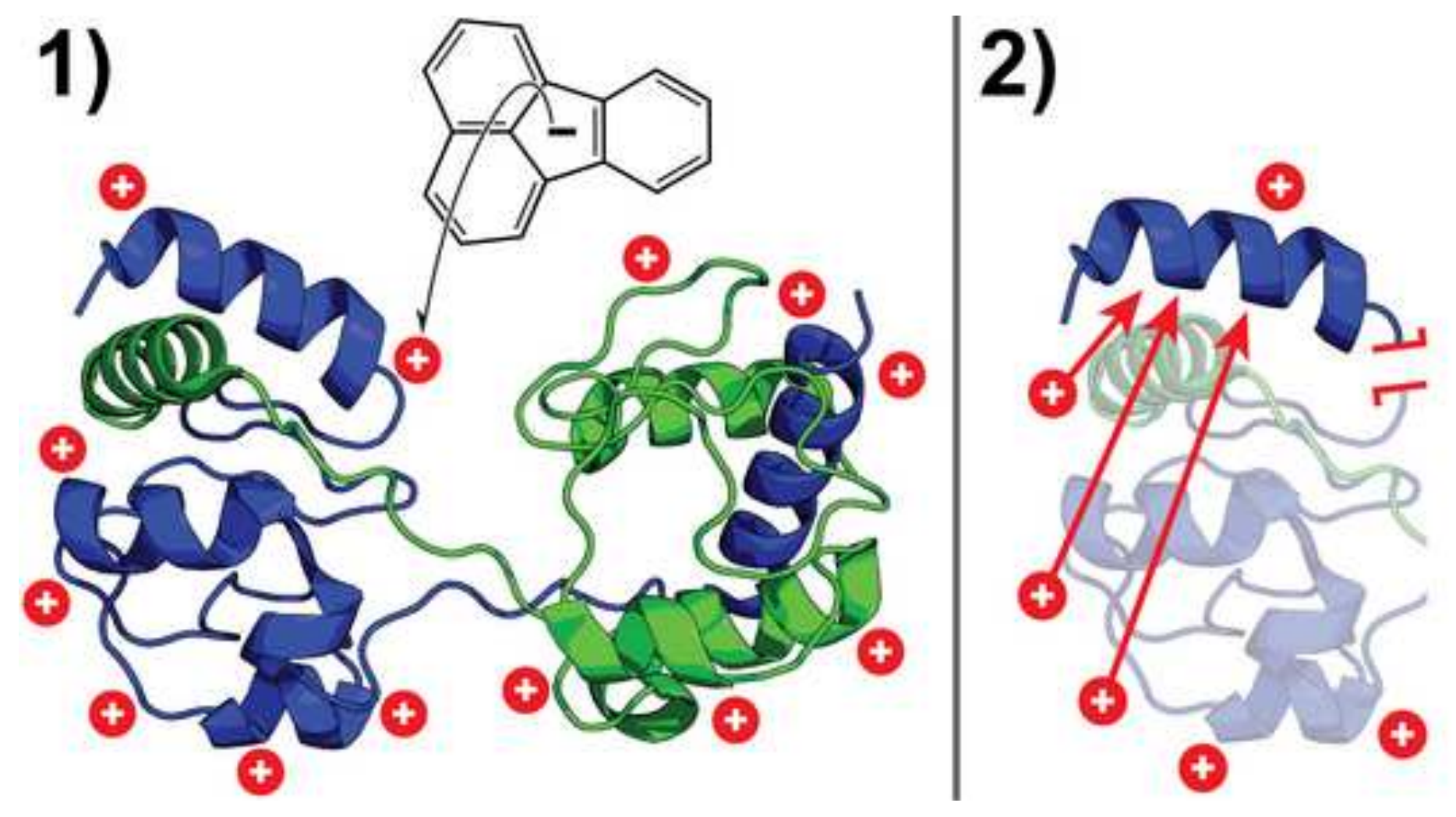

3) ำํํㅇ

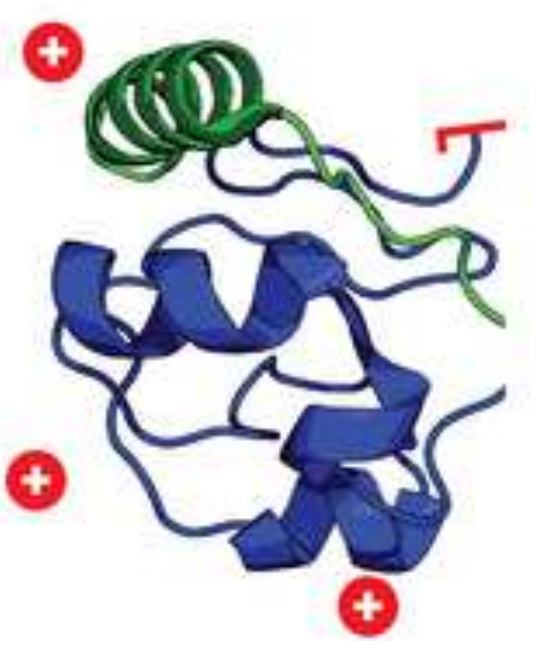

